\section{Comment on "Quantum Theory of Secondary Emission in Optically Excited Semiconductor Quantum Wells"}

In a recent Letter, Kira et al. [1] propose a fully quantum mechanical theory for the secondary emission (SE) of a quantum well $(\mathrm{QW})$, assuming that the $\mathrm{QW}$ is free of disorder, and that SE originates from the interaction between excitons in the system. The authors ask if "disorder is necessary to explain coherent signatures in the SE." They claim that their "microscopic theory reproduces many of the experimentally observed effects, showing that the SE exhibits intrinsic coherent characteristics...." In this Comment we show that the SE calculated by Kira et al. is incoherent and nonlinear, and therefore inadequate for SE experiments performed under low-excitation conditions [2-8]. Such experiments are in agreement with an explanation in terms of disorder-induced resonant Rayleigh scattering (RRS) and have indeed given clear evidence for the following: (i) The temporal coherence of the $\mathrm{SE}$ at early times. (ii) A fully linear relation between emission intensity and excitation density over 3 orders of magnitude. (iii) The temporal profile depends only on statistical properties of the disordered potential.

On the other hand, the new theoretical formalism by Kira et al. combining semiconductor Bloch equations and field quantization is of largest importance for the dynamics of temporally incoherent resonant QW luminescence [3,9].

The term "temporal coherence" of SE has received very lately a clear definition that is experimentally verifiable. The coherent part of SE produces interferences with a replica of the exciting laser pulse $[5,8]$ and present speckles [7]; because the quantum average of the electric field operator in the SE direction is nonzero, $\left\langle E_{\mathbf{q}}\right\rangle \equiv\left\langle b_{\mathbf{q}}+b_{\mathbf{q}}^{\dagger}\right\rangle \neq$ 0 . Experiments show that this average electric field makes up $25 \%$ [5] or up to $50 \%$ [8] of the total intensity of the SE. Fluctuations of the electric field operator are much too weak to explain these experimental findings. The calculations by Kira et al. [1] use explicitly the fact that only in the specular directions is $\left\langle E_{\mathbf{q}}\right\rangle \neq 0$. For the scattered directions only the correlation functions $\left\langle b_{q_{z}, \mathbf{q}_{\|}}^{\dagger} h_{-\mathbf{k}} e_{\mathbf{k}+\mathbf{q}_{\|}}\right\rangle$ are nonzero. The calculated SE is therefore mainly incoherent as it does not carry a phase that could produce strong enough interferences, which would be quantitatively in agreement with the experiments $[5,8]$.

Another important property of the coherent SE is the linear dependence on the excitation density, which has been explicitly reported $[5,6,10]$. The early SE calculated by Kira et al. shows a nonlinear dependence of the peak intensity and density-dependent rise time (see Fig. 1 in [1]).
Nonlinearity is also explicit in their two pulse calculations in which "the two pulse excitation for $\Phi=0$ leads to an enhancement of the emission intensity by more than an order of magnitude" in comparison to the single-pulse excitation. Experiments on SE excited by two pulses show that the enhancement factor is never larger than $4[4,10]$, as predicted by linear theories [11].

Finally, the physical origin of the "pulse replica" found at $t \simeq 500$ fs needs to be clarified. If the pulse replica is due to exciton-exciton interaction, we would expect that the position of the peak depends on the exciton density, something that does not seem to happen in the work of Kira et al. [1] (Fig. 1 in [1]).

Our conclusions are as follows: (i) the interactioninduced SE [1] is incoherent and nonlinear. (ii) It is therefore incompatible with the experimentally observed coherent and linear SE. (iii) The SE calculated by Kira et al. is more appropriate and of highest value for the description of incoherent luminescence dynamics due to exciton-exciton interaction.

J. Fernández-Rossier, ${ }^{1, *}$ S. Haacke, ${ }^{2}$ and B. Deveaud ${ }^{2}$

${ }^{1}$ Departamento de Física Teórica de la Materia Condensada

Universidad Autónoma de Madrid

Cantoblanco, 28049 Madrid, Spain

${ }^{2}$ Physics Department, IMO

Swiss Federal Institute of Technology Lausanne

CH-105 Lausanne, EPFL, Switzerland

Received 14 July 1999

PACS numbers: 78.66.-w, 42.25.Kb, 42.50.Dv

* Present address: Physics Department, University of California San Diego, 9500 Gilman Drive, La Jolla, CA 92092.

[1] M. Kira, F. Jahnke, and S. W. Koch, Phys. Rev. Lett. 82, 3544 (1999).

[2] M. Gurioli et al., Phys. Rev. Lett. 78, 3205 (1997).

[3] S. Haacke et al., Phys. Rev. Lett. 78, 2228 (1997).

[4] M. Woerner and J. Shah, Phys. Rev. Lett. 81, 4208 (1998).

[5] D. Birkedal and J. Shah, Phys. Rev. Lett. 81, 2372 (1998).

[6] S. Haacke, et al., in OSA Trends in Optics and Photonics, edited by D. Citrin (Optical Society of America, Washington, DC, 1998), Vol. 18, p. 64.

[7] W. Langbein, J. Hvam, and R. Zimmermann, Phys. Rev. Lett. 82, 1040 (1999).

[8] S. Haacke et al., Phys. Rev. B 61, 5109 (2000).

[9] H. Wang et al., Phys. Rev. Lett. 74, 3065 (1995).

[10] N. Garro (private communication); N. Garro et al., Phys. Rev. B 60, 4497 (1999).

[11] J. Fernández-Rossier, C. Tejedor, and R. Merlin, J. Phys. Condens. Matter 11, 6013 (1999). 